\title{
A Synchronization Technique for Optical PPM Signals
}

\author{
V. A. Vilnrotter, E. R. Rodemich and H. H. Tan \\ Communications Systems Research Section
}

\begin{abstract}
A technique for maintaining synchronization between optical PPM pulses and a receiver clock by means of a delay-tracking loop is described and analyzed. The tracking loop is driven by a doubly stochastic Poisson process that contains information about the location of the desired slot boundaries. The slot boundaries are subject to slowly varying random delays that are ultimately tracked by the loop. The concept of fractional rms delay error is introduced to quantify the effects of signal and background induced shot noise on the performance of the delay-tracking loop.
\end{abstract}

\section{Introduction}

Optical pulse-position modulation (PPM) is an accepted signaling format for the transmission of information over optical channels. With this coding technique, $L$ bits of information are encoded onto one of $M=2^{L}$ PPM words by establishing a one-to-one correspondence between the possible states of $L$ binary digits and the location of an optical pulse among $M$ possible slots. If the slot duration is $\tau$ seconds, then each PPM word requires $M \tau$ seconds for transmission. For a direct-detection system, maximum likelihood decoding consists of counting the number of photons in each slot and selecting the PPM word corresponding to the greatest count. To accomplish this, however, requires accurate synchronization between the received PPM slot boundaries and the counting boundaries defined by the receiver clock. Synchronization for binary PPM optical signals by means of "early-late" gates and decision-directed feedback has been considered previously in the literature (Refs. 1,2 ). Here we consider a novel technique for synchronizing $M$-ary PPM symbols without the use of decision-directed feedback, thus effectively removing the dependence of the synchronization subsystem on decoder performance. The description and analysis of this tracking system is the subject of the following sections.

\section{Tracking System Description and Analysis}

The slot synchronization technique described here relies on the addition of a small "dead time" before and after each optical pulse, in order to derive an error signal proportional to the delay error that may exist between the received slot boundaries and the receiver clock. (Decoder performance is not affected if the pulse intensity at the transmitter can be increased to maintain constant average pulse count at the receiver.) Thus, $\tau=\tau_{p}+2 \tau_{d}$, where $\tau_{p}$ is the duration of the optical pulse and $\tau_{d}$ the duration of each "dead-time" interval.

The detector output can be modeled as a doubly stochastic Poisson process, represented by a sequence of randomly occurring unit impulses whose average rate at any given time depends on the total signal and background power collected by the 
receiver. Consider the case where the receiver clock is synchronized with the received optical data (we do not address the acquisition problem here). Doppler effects and transmitter or receiver clock instabilities tend to introduce random delay components between the received data and the receiver clock which, if uncorrected, may eventually cause loss of synchronization. Assuming that the tracking system maintains synchronism between the received data and the receiver clock, transmitter clock instabilities and Doppler-induced rate changes can be modeled in terms of the delay process $\Delta \tau=$ $\Delta \tau_{c t}+\Delta \tau_{D}$, whose components are transmitter clock drift and Doppler-induced delay, respectively. Typically, $\Delta \tau$ is a slowly varying function of time that can be treated as a constant over a great many PPM words.

A block diagram of the delay-tracking loop is shown in Fig. 1. The detector output process $x(t ; \Delta \tau)$, which contains information about the location of the transmitted datapulses, serves as input to the tracking loop. This is a doubly stochastic Poisson process with random rate $\lambda(t ; \Delta \tau)=\lambda_{s}(t ; \Delta \tau)$ $+n_{b}$, where $\lambda_{s}(t ; \Delta \tau)$ is a random rate due to the signal while $n_{b}$ is a constant average count rate due to multimode background radiation. The receiver clock generates a squarewave $g\left(t ; \widehat{\Delta \tau}_{n}\right)$ of nominal period $\tau$ and noisy delay estimate $\widehat{\Delta \tau_{n}}=$ $\widehat{\Delta \tau}+\Delta \tau_{c r}$. The components of $\widehat{\Delta \tau_{n}}$ are the loop's estimate of the received delay $\Delta r$, and an independent drift component $\Delta \tau_{c r}$ due to instabilities within the receiver clock. The input to the loop filter is the product of the detector output process and clock waveform. Defining the delay error as $\Delta \tau_{\epsilon}=\Delta \tau-$ $\widehat{\Delta \tau_{n}}$, this process can be represented as

$$
y\left(t ; \Delta \tau_{\epsilon}\right)=x(t ; \Delta \tau) g\left(t ; \widehat{\Delta \tau_{n}}\right)
$$

where the notation implies that the product contains information about the delay error $\Delta \tau_{\epsilon}$. Indeed, for $M$-ary PPM symbols with dead-time, the time averaged mean value given $\Delta \tau$ and $\widehat{\Delta \tau_{n}}$, clearly depends on $\Delta \tau_{\epsilon}$ :

$$
\begin{aligned}
\bar{y}\left(\Delta \tau_{\epsilon}\right) \quad & \Delta \lim _{T \rightarrow \infty} \frac{1}{2 T} \int_{-T}^{T} E\left\{x(t ; \Delta \tau) g\left(t ; \widehat{\Delta \tau_{n}}\right\} d t\right. \\
& =\lim _{T \rightarrow \infty} \frac{1}{2 T} \int_{-T}^{T} \lambda(t ; \Delta \tau) g\left(t ; \widehat{\Delta \tau_{n}}\right) d t \\
& =\frac{2 n_{s}}{M \tau} \Delta \tau_{\epsilon}=\alpha \Delta \tau_{\epsilon} ;\left|\Delta \tau_{\epsilon}\right| \leqslant \delta \\
\delta & \Delta \min \left(\tau_{d}, \frac{\tau}{2}-\tau_{d}\right)
\end{aligned}
$$

where $2 \delta$ is the extent of the linear range, $E$ is the expectation operator, $n_{s}$ is the Poisson rate due to signal when a pulse is present, and $\alpha=2 n_{s} / M \tau$. Note that $\bar{y}\left(\Delta \tau_{\epsilon}\right)$ does not depend on the background induced count rate since the time average of $n_{b} g\left(t ; \Delta \tau_{n}\right)$ over an integral number of periods is zero. Figure 2 illustrates the behavior of $\bar{y}\left(\Delta \tau_{\epsilon}\right)$ as a function of $\Delta \tau_{\epsilon}$ over the interval $\left|\Delta \tau_{\epsilon}\right| \leqslant \tau / 2$, assuming that $\tau_{d}<\tau / 4$.

It is convenient to decompose $y\left(t ; \Delta \tau_{\epsilon}\right)$ as

$$
y\left(t ; \Delta \tau_{\epsilon}\right)=\bar{y}\left(\Delta \tau_{\epsilon}\right)+n\left(t ; \Delta \tau_{\epsilon}\right)
$$

where $n\left(t ; \Delta \tau_{\epsilon}\right) \triangleq y\left(t ; \Delta \tau_{\epsilon}\right)-\bar{y}\left(\Delta \tau_{\epsilon}\right)$ is viewed as an additive noise process, containing the conditioning parameter $\Delta \tau_{\epsilon}$. The power spectral density of this noise process conditioned on $\Delta \tau_{\epsilon}, S_{n}\left(\omega ; \Delta \tau_{\epsilon}\right)$, is derived in Appendix A. There it is shown that for $\omega=0$ the noise power spectral density does not depend on $\Delta \tau_{\epsilon}$, but takes the value $\left(\widetilde{n}_{s}+n_{b}\right)$, where

$$
\tilde{n}_{s} \triangleq n_{s} \tau_{p} / M \tau
$$

is the average Poisson count rate generated by the signal. Thus, in a suitably narrow band of frequencies around the origin, the power spectral density of the equivalent noise process may be approximated as

$$
S_{n}(\omega) \simeq \widetilde{n}_{s}+n_{b} ;|\omega| \ll \frac{2 \pi}{M \tau}
$$

If $\Delta \tau_{\epsilon}$ varies slowly enough to be considered constant over a great many PPM symbols, then $\bar{y}\left(\Delta \tau_{\epsilon}\right)$ and $S_{n}\left(\omega ; \Delta \tau_{\epsilon}\right)$ may be approximated by replacing the infinite limits in their defining equations by finite values that encompass regions of constant $\Delta \tau_{\epsilon}$. In that case, we conclude that for frequencies near the origin, the process $y\left(t ; \Delta \tau_{\epsilon}\right)$ behaves as though it were composed of a slowly varying term proportional to the delay error; plus an independent additive white noise process $n(t)$ characterized by the spectral level $\left(\tilde{n}_{s}+n_{b}\right)$, as defined in equation (4).

Assuming that $\left|\Delta \tau_{\epsilon}\right| \ll \delta$, the operation of the tracking loop can be described by means of the linear loop model shown in Fig. 3 (Ref. 3). Using the differential operator $p \triangleq d / d t$, loop operation can be expressed in terms of the equation

$$
\begin{aligned}
& \Delta \hat{\tau}=\Delta \tau_{c}+\Delta \tau_{D}-\Delta \tau_{\epsilon}=\frac{G}{P} F(p)\left[\alpha \Delta \tau_{\epsilon}+n\right] \\
& \Delta \tau_{c} \Delta \Delta \tau_{c t}-\Delta \tau_{c r}
\end{aligned}
$$

where $G$ is the gain of the voltage-controlled clock and $n=$ $n\left(t ; \Delta \tau_{\epsilon}\right)$ as in Eq. (3) (the time index has been deleted for the sake of notational simplicity). Solving for $\Delta \tau_{\epsilon}$ yields 


$$
\begin{gathered}
\Delta \tau_{\epsilon}=[1-H(p)]\left(\Delta \tau_{c}+\Delta \tau_{D}\right)-H(p) \frac{n}{\alpha} \\
H(p) \triangleq \alpha G F(p) /(p+\alpha G F(p))
\end{gathered}
$$

The tracking system should be designed to track $\Delta \tau_{c}$ and $\Delta \tau_{D}$ accurately, in which case their contributions to $\Delta \tau_{\epsilon}$ can be ignored. Under this condition the steady-state delay-error is due entirely to the equivalent noise process $n(t)$. Assuming that $|H(j \omega)|^{2}$ takes on significant values only in an interval $|\omega| \ll 2 \pi / M \tau$, the variance of the delay-error becomes

$$
\begin{aligned}
\sigma_{\epsilon}^{2} \Delta \operatorname{var}\left(\Delta \tau_{\epsilon}\right) & =\frac{1}{2 \pi \alpha^{2}} \int_{-\infty}^{\infty}|H(j \omega)|^{2} S_{n}(\omega) d \omega \\
& \simeq \frac{2 B_{L}}{\alpha^{2}} S_{n}(0)
\end{aligned}
$$

where $B_{L}$ is the one-sided loop bandwidth defined by

$$
2 B_{L}=\frac{1}{2 \pi} \int_{-\infty}^{\infty}|H(j \omega)|^{2} d \omega
$$

Substituting for $\alpha$ and $S_{n}(0)$ in equation (7) yields

$$
\sigma_{\epsilon}^{2}=\frac{B_{L} M^{2} \tau^{2}}{2 n_{s}^{2}}\left(\widetilde{n_{s}}+n_{b}\right)=\frac{B_{L} \tau_{p}^{2}}{2 \widetilde{n}_{s}^{2}}\left(\widetilde{n_{s}}+n_{b}\right)
$$

From equation (9) it is clear that the fractional rms delay error $\left(\sigma_{\epsilon} / \tau_{p}\right)$ depends only on $B_{L}, \widetilde{n}_{s}$ and $n_{b}$, and is independent of $\tau_{p}$ for any fixed value of $\widetilde{n}_{s}$ :

$$
\left(\frac{\sigma_{\epsilon}}{\tau_{p}}\right)=\sqrt{\frac{B_{L}}{2 \widetilde{n}_{s}}\left(1+\frac{n_{b}}{\widetilde{n}_{s}}\right)}
$$

Note that even in the absence of background radiation $\left(\sigma_{\epsilon} / \tau_{p}\right)$ does not become arbitrarily small, but approaches its quantum-limited value $\sqrt{B_{L} / 2 \widetilde{n}_{s}}$.

It is apparent from equations (6b) and (8) that $B_{L}$ depends only on the loop filter transfer function $F(p)$ and the product $\alpha G$. Since $G$ is independent of the other system parameters, constant loop bandwidth can be maintained by adjusting $G$ so as to keep $\alpha G$ constant. Thus, $B_{L}$ can be held fixed even as $\widetilde{n}_{s}=\alpha \tau_{p} / 2$ varies. The behavior of $\left(\sigma_{\epsilon} / \tau_{p}\right)$ as a function of $\tilde{n}_{s}$ for various loop bandwidths and background rates is shown in Fig. 4.
Other meaningful fractional rms delay errors may also be defined in terms of $\left(\sigma_{\epsilon} / \tau_{p}\right)$, namely

$$
\begin{aligned}
& \left(\frac{\sigma_{\epsilon}}{\tau_{d}}\right)=\left(\frac{\tau}{\tau_{d}}-2\right)\left(\frac{\sigma_{\epsilon}}{\tau_{p}}\right) ; 0<\tau_{d} \leqslant \frac{\tau}{2} \\
& \left(\frac{\sigma_{\epsilon}}{\tau}\right)=\left(1-\frac{2 \tau_{d}}{\tau}\right)\left(\frac{\sigma_{\epsilon}}{\tau_{p}}\right) ; 0<\tau_{d} \leqslant \frac{\tau}{2}
\end{aligned}
$$

and using Eq. (2b),

$$
\left(\frac{\sigma_{\epsilon}}{\delta}\right)=\left\{\begin{array}{l}
\left(\frac{\sigma_{\epsilon}}{\tau_{d}}\right) ; \quad 0<\tau_{d} \leqslant \frac{\tau}{4} \\
2\left(\frac{\sigma_{\epsilon}}{\tau_{p}}\right) ; \frac{\tau}{4}<\tau_{d} \leqslant \frac{\tau}{2}
\end{array}\right.
$$

It should be emphasized that the above equations are meaningful only if the inequality $\left(\sigma_{\epsilon} / \delta\right) \ll 1$ is satisfied. Assuming this to be the case, Eqs. (11a) and (11c) show that $\left(\sigma_{\epsilon} / \delta\right)$ is a monotone decreasing function of $\tau_{d}$ in the interval $0 \stackrel{\epsilon}{<} \tau_{d} \leqslant$ $\tau / 4$, while maintaining a constant minimum value of $2\left(\sigma_{\epsilon} / \tau_{p}\right)$ over the interval $\tau / 4<\tau_{d}<\tau / 2$.

In typical applications, the loop bandwidth $B_{L}$ is chosen large enough to exceed the significant spectral components of $\Delta \tau_{c}$ and $\Delta \tau_{D}$, thus allowing the loop to track these quantities accurately. The signal-set dimension $M$ and slot-width $\tau$ are generally determined on the basis of communications requirements, while $n_{b}$ depends on the background environment and on the particular set of spatial and frequency predetection filters employed. In order to achieve the desired decoder error probability, some minimum average signal pulse count $n_{s} \tau_{p}$ must be maintained, even in the absence of synchronization errors. Under the condition $\left(\sigma_{\epsilon} / \delta\right) \ll 1$, random delay errors should have little effect on decoder performance if the decoder counts photons over the entire synchronous $\tau$-second time interval. Thus, a value of $\left(\sigma_{\epsilon} / \delta\right)$ can generally be found to guarantee negligible decoder performance degradation while simultaneously assuring linear loop operation: let this value be denoted by $\gamma$. Again using Eqs. (11a) and (11c), a reasonable value for $\tau_{d}$ can be determined by calculating $\left(\sigma_{\epsilon} / \tau_{p}\right)$ from the given system parameters, and comparing it to $\gamma / 2$. If $\left(\sigma_{\epsilon} / \tau_{p}\right) \leqslant \gamma / 2$, then let

$$
\tau_{d}=\tau\left[2+\left(\gamma /\left(\frac{\sigma_{\epsilon}}{\tau_{p}}\right)\right)\right]^{-1}
$$


However, if $\left(\sigma_{\epsilon} / \tau_{p}\right)>\gamma / 2$, then the desired value of $\left(\sigma_{\epsilon} / \delta\right)$ cannot be realized with the initial set of system parameters, and hence at least one of the original parameters must be altered to accommodate tracking-loop requirements. Generally, the tracking loop will perform well, with dead-times substantially less than $\tau / 4$ seconds, when operating in high data-rate narrow band communication systems where a suitably small value of $\left(\sigma_{\epsilon} / \tau_{p}\right)$ can be achieved.

\section{Concluding Remarks}

A technique has been developed for maintaining synchronization between a PPM-modulated optical data-stream and a receiver clock. This technique relies on the addition of a small "dead time" interval before and after each possible pulse location to derive an electrical signal proportional to the delay error between the received slot boundaries and the receiver clock. It was argued that in applications where only spectral components near the origin are of interest, and where the delay error remains essentially constant over a great many
PPM symbols, the driving process could be approximated by a slowly varying term proportional to the delay error, plus an effective additive noise process due to the inherent randomness of the detected optical fields. Based on the above approximations, the variance of the delay error due to both signal and background radiation was derived, assuming a linear loop that effectively tracked out the slowly varying delay error components. We observed that since linear loop operation typically implied rms delay errors on the order of a small fraction of the linear region, decoder performance should not be significantly affected if the decoder counts photons over the entire $\tau$-second slot time. It was shown that in applications where this is true, a simple algorithm can often be employed to find "good" design values for the dead-time interval in terms of the given system parameters, and a quantity the designer selects to simultaneously ensure linear loop operation and acceptable decoder performance. However, a precise determination of the dead-time interval (taking into account decoder performance degradation and possible non-linear effects within the loop) is beyond the scope and interest of this article.

\section{References}

1. Gagliardi, R. M., "Synchronization Using Pulse Edge Tracking in Optical Pulse-Position Modulated Communication Systems," IEEE Transactions on Communications, Oct. 1974. Vol. Com-22. No. 10, pp. 1693-1702.

2. Gagliardi, R. M. and Karp, S., Optical Communications, Wiley, N.Y., 1976.

3. Lindsey, W. C., Synchronization Systems in Communications and Control, Prentice Hall, N.J., 1972, Chapt. 4. 


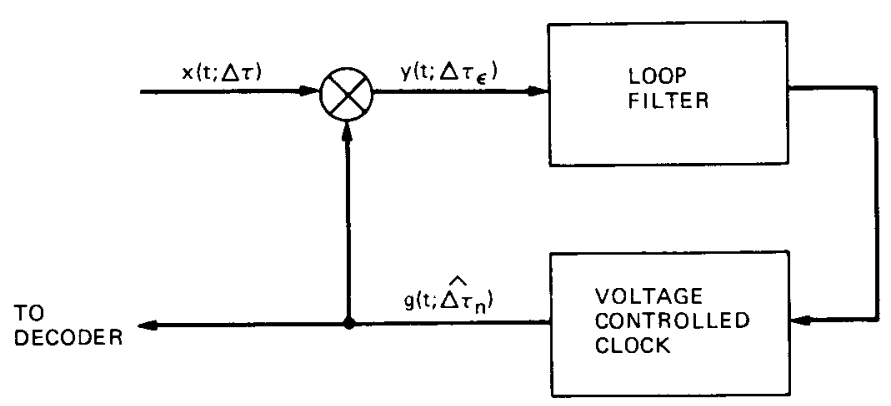

Fig. 1. Tracking loop block diagram

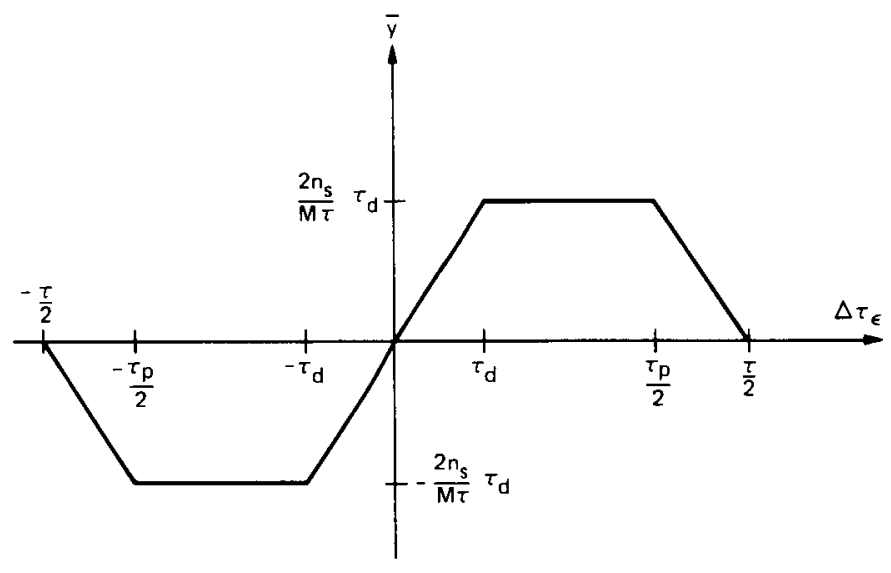

Fig. 2. Time averaged mean value as a function of delay error $\left(\tau_{d}<\tau / 4\right)$

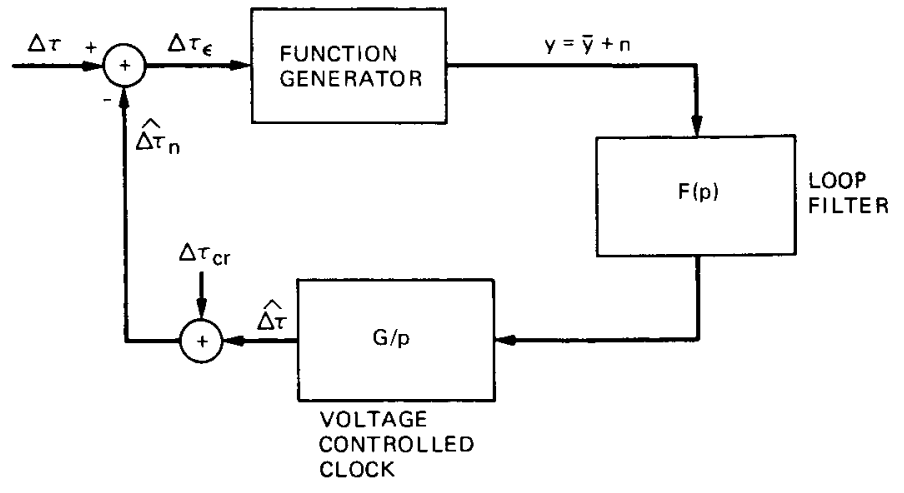

Fig. 3. Linear loop model

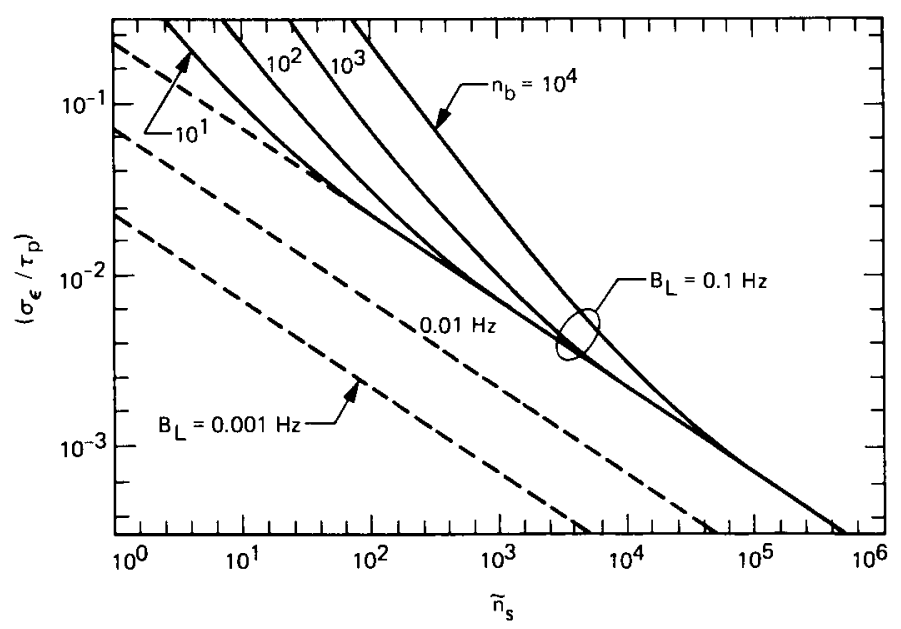

Fig. 4. Fractional rms delay error $\left(\sigma_{\epsilon} / \tau_{p}\right)$ as a function of average signal count for various background rates and loop bandwidths 


\section{Appendix A}

The power spectral density of $n\left(t ; \Delta \tau_{\epsilon}\right)$ can be derived using the results of Appendix B. Assume that $\Delta \tau_{\epsilon}$ is held fixed. Since $n\left(t ; \Delta \tau_{\epsilon}\right)=y\left(t ; \Delta \tau_{\epsilon}\right)-\bar{y}\left(\Delta \tau_{\epsilon}\right)$, it follows that

$$
S_{n}\left(\omega ; \Delta \tau_{\epsilon}\right)=S_{y}\left(\omega ; \Delta \tau_{\epsilon}\right)-S_{\bar{y}}\left(\omega ; \Delta \tau_{\epsilon}\right)
$$

where

$$
S_{\bar{y}}\left(\omega ; \Delta \tau_{\epsilon}\right)=\left(\frac{2 n_{s} \Delta \tau_{\epsilon}}{M \tau}\right)^{2} 2 \pi \delta(\omega)
$$

Consider the case where the detector observes signal plus multimode background noise fields. Thus, $\lambda(t ; \Delta \tau)=\lambda_{s}(t ; \Delta \tau)+$ $n_{b}$ where $\lambda_{s}(t ; \Delta \tau)$ is the random rate function associated with the signal while $n_{b}$ is a constant average count rate contributed by the background fields. The square-wave generated by the receiver clock is denoted by $g\left(t ; \widehat{\Delta \tau_{n}}\right)$ as before. For $M$-ary PPM with dead-time the product $\lambda(t ; \Delta \tau) g\left(t ; \widetilde{\Delta \tau_{n}}\right)$ can be modeled over the interval $[-T, T]$ as

$$
\begin{aligned}
\lambda(t ; \Delta \tau) g\left(t ; \widehat{\Delta \tau_{n}}\right)= & \sum_{i=-K}^{K} p_{s}\left(t-\left(i M \tau+\ell_{i} \tau-\frac{M \tau}{2}\right)\right) \\
& +\sum_{i=-K}^{K} \sum_{k=0}^{M-1} p_{b}\left(t-\left(i M \tau+k \tau-\frac{M \tau}{2}\right)\right)
\end{aligned}
$$

where $K=(T / M \tau)-1 / 2, \tau$ is the slot duration, and $M \tau$ the duration of a PPM word. Elements of the set $\left\{\ell_{i}\right\}$ are independent random integers taking on values from 0 to $(M-1)$ with equal probability. Given $\Delta \tau_{\epsilon}, p(t)$ and $p_{b}(t)$ are defined as

$$
\begin{aligned}
& p_{s}(t)=\left\{\begin{array}{l}
0 ; 0 \leqslant t<\tau_{d}, \tau-\tau_{d} \leqslant t<\tau \\
n_{s} ; \tau_{d} \leqslant t<\frac{\tau}{2}+\Delta \tau_{\epsilon} \\
-n_{s} ; \frac{\tau}{2}+\Delta \tau_{\epsilon} \leqslant t<\tau-\tau_{d}
\end{array}\right. \\
& p_{b}(t)=\left\{\begin{array}{l}
n_{b} ; \Delta \tau_{\epsilon} \leqslant t<\frac{\tau}{2}+\Delta \tau_{\epsilon} \\
-n_{b} ; 0 \leqslant t<\Delta \tau_{\epsilon}, \frac{\tau}{2}+\Delta \tau_{\epsilon} \leqslant t<\tau
\end{array}\right.
\end{aligned}
$$

With $P_{s}(\omega) \triangleq F\left\{p_{s}(t)\right\}$ and $P_{b}(\omega) \triangleq F\left\{p_{b}(t)\right\}$, the Fourier transform of $\lambda(t ; \Delta \tau) g\left(t, \Delta \tau_{n}\right)$ restricted to the interval $[-T, T)$, becomes

$$
\begin{aligned}
F_{T}\left\{\lambda(t ; \Delta \tau) g\left(t ; \widehat{\Delta \tau_{n}}\right)\right\}= & \left\{P_{s}(\omega) \sum_{i=-K}^{K} \mathrm{e}^{-j \omega\left(i M \tau+\ell_{i} \tau\right)}\right. \\
& \left.+M P_{b}(\omega) \gamma(\omega)\right\} \mathrm{e}^{j \omega M \tau / 2}
\end{aligned}
$$

where

$$
\gamma(\omega)=\sum_{i=-K}^{K} \mathrm{e}^{-j \omega i M \tau} E\left(\mathrm{e}^{-j \omega \ell_{i} \tau}\right)
$$$$
=\mathrm{e}^{-(1 / 2) j \omega(M-1) \tau}\left[\frac{\sin \left(\left(K+\frac{1}{2}\right) \omega M \tau\right)}{M \sin \left(\frac{1}{2} \omega \tau\right)}\right]
$$

$$
\left.\left.P_{s}(\omega)=\frac{n_{s}}{j \omega}\left[\mathrm{e}^{-j \omega \tau} d+\mathrm{e}^{-j \omega(\tau-\tau} d\right)-2 \mathrm{e}^{-j \omega(\tau / 2+\Delta \tau} \epsilon\right)\right]
$$

and

$$
P_{b}(\omega)=\frac{n_{b}}{j \omega}\left[2 \mathrm{e}^{-j \omega \Delta \tau} \epsilon+\mathrm{e}^{-j \omega \tau}-2 \mathrm{e}^{-j \omega(\tau / 2+\Delta \tau} \epsilon^{\prime}-1\right]
$$

Recognizing that

$$
\sum_{i=-K}^{K} \sum_{n=-K}^{K} \mathrm{e}^{-j \omega(i-n) M \tau} E\left\{\mathrm{e}^{-j \omega\left(\ell_{i}-\ell_{n}\right) \tau}\right\}
$$

$$
=|\gamma(\omega)|^{2}+(2 K+1)\left[1-\left(\frac{\sin \left(\frac{1}{2} \omega M \tau\right)}{M \sin \left(\frac{1}{2} \omega \tau\right)}\right)^{2}\right]
$$


and making use of Eq. (A-4), it follows that

$$
\begin{aligned}
& \frac{1}{2 T} E\left\{\left|F_{T}\{\lambda g\}\right|^{2}\right\}=\frac{1}{2 T}\left\{\left|P_{s}(\omega)+M P_{b}(\omega)\right|^{2}|\gamma(\omega)|^{2}\right. \\
& \left.+\left|P_{s}(\omega)\right|^{2}(2 K+1)\left[1-\left(\frac{\sin \left(\frac{1}{2} \omega M \tau\right)}{M \sin \left(\frac{1}{2} \omega \tau\right)}\right)\right]\right\}
\end{aligned}
$$

Note that

$$
\lim _{K \rightarrow \infty} \frac{|\gamma(\omega)|^{2}}{(2 K+1)}=\frac{2 \pi}{M \tau} \sum_{m=-\infty}^{\infty} \delta\left(\omega-\frac{2 \pi m}{\tau}\right)
$$

To show this we first observe that $\gamma(\omega)$ is a periodic function of $\omega$ with period $2 \pi / \tau$. Over any interval $I$ that does not contain the points $\omega_{m}=2 \pi m / \tau$, the denominator

\section{$M \sin (\omega \tau / 2)$}

is bounded away from zero, hence $|\gamma(\omega)|^{2}$ is bounded, and

$$
\lim _{K \rightarrow \infty} \frac{|\gamma(\omega)|^{2}}{(2 K+1)}=0 ; \quad \omega_{m} \notin I
$$

Thus, the integral of $|\gamma(\omega)|^{2} /(2 K+1)$ vanishes everywhere except within arbitrarily small neighborhoods around the points $\omega_{m}$ as $K$ grows without bound. However, integration over any complete cycle yields

$$
\begin{aligned}
\int_{2 \pi(m-1 / 2) / \tau}^{2 \pi(m+1 / 2) / \tau} d \omega|\gamma(\omega)|^{2} /(2 K+1) & =\frac{1}{M^{2}(2 K+1)} \sum_{i=-K}^{K} \sum_{\ell=0}^{M-1} \frac{2 \pi}{\tau} \\
& =\frac{2 \pi}{M \tau}
\end{aligned}
$$

independent of $K$. Therefore, this is the value of the integral over arbitrarily small neighborhoods around each point $\omega_{m}$ as $K$ approaches infinity.

We also make the useful observations that $P_{b}(0)=0$, $\left|P_{s}(0)\right|^{2}=4 n_{s}^{2} \Delta \tau_{\epsilon}^{2}$, and

$$
\begin{gathered}
\lim _{T \rightarrow \infty} \frac{1}{2 T} \int_{-T}^{T} E\left\{\left|g\left(t: \widehat{\Delta \tau_{n}}\right)\right|^{2} \lambda(t ; \Delta \tau) d t\right. \\
=\lim _{T \rightarrow \infty} \frac{1}{2 T} \int_{-T}^{T} \lambda(t ; \Delta \tau) d t \\
=\tilde{n}_{s}+n_{b}
\end{gathered}
$$

where

$$
\tilde{n}_{s}=n_{s} \tau_{p} / M \tau \text { and } \quad \mid g\left(\left.t \cdot \widehat{\Delta \tau}_{n}\right|^{2}=1\right.
$$

Making use of the fact that $2 T=(2 K+1) M \tau$, substituting the above terms into equation (B-7) and using Eq. (A-1) finally yields

$$
S_{n}\left(\omega ; \Delta \tau_{\epsilon}\right)=\widetilde{n}_{s}+n_{b}+\frac{\left|P_{s}(\omega)\right|^{2}}{M \tau}\left[1-\left(\frac{\sin \left(\frac{1}{2} \omega M \tau\right)}{M \sin \left(\frac{1}{2} \omega \tau\right)}\right)^{2}\right]
$$

$$
+\left|P_{s}(\omega)+M P_{b}(\omega)\right|^{2} \frac{2 \pi}{M^{2} \tau^{2}} \sum_{\substack{m=-\infty \\ m \neq 0}}^{\infty} \delta\left(\omega-\frac{2 \pi m}{\tau}\right)
$$

Thus, the spectral density of the equivalent noise process consists of continuous and impulsive terms. The impulses occur at integer multiples of the radian frequency $2 \pi / \tau$; however, there is no impulse at the origin since the contribution of $\bar{y}$ was subtracted out. The value of the continuous part at the origin is

$$
S_{n}\left(0 ; \Delta \tau_{\epsilon}\right)=\tilde{n}_{s}+n_{b}
$$

independent of $\Delta \tau_{\epsilon}$. Therefore, this must be the value of the unconditioned spectral density as well:

$$
S_{n}(0)=E_{\Delta \tau_{\epsilon}}\left\{S_{n}\left(0 ; \Delta \tau_{\epsilon}\right)\right\}=\widetilde{n}_{s}+n_{b}
$$

Since for any $\left|\Delta \tau_{\epsilon}\right| \leqslant \delta$, the continuous part of Eq. (A-6) begins to deviate significantly from its zero-frequency value only as $\omega$ approaches values on the order of $2 \pi / M \tau$, it can be argued that in a suitably narrow band of frequencies around the origin (namely, $|\omega| \ll 2 \pi / M \tau$ ) the spectral density of the equivalent noise process may be approximated by its zerofrequency value. 


\section{Appendix B}

The power spectral density of the product of a doubly stochastic Poisson process and a deterministic function is derived. Let

$$
x(t)=\sum_{\ell} \delta\left(t-t_{\ell}\right)
$$

be a sample function of a doubly stochastic Poisson process with random rate $\lambda(t)$. Let $g(t)$ be a deterministic function and form the product $y(t)=g(t) x(t)$. Define the power spectral density of $y(t), S_{y}(\omega)$, as in Ref. 2 (Chapter 4):

$$
S_{y}(\omega)=\lim _{T \rightarrow \infty} \frac{1}{2 T} E\left|Y_{T}(\omega)\right|^{2}
$$

where

$$
Y_{T}(\omega) \triangleq \int_{-T}^{T} \mathrm{e}^{-j \omega t} y(t) d t=F_{T}\{y(t)\}
$$

is the Fourier transform of $g(t)$ restricted to the interval $[-T, T)$. Using Eq. (B-1) yields

$$
\begin{gathered}
Y_{T}(\omega)=\sum_{\ell} g\left(t_{\ell}\right) \mathrm{e}^{-j \omega t_{\ell}} ; t \epsilon[-T, T) \\
\left|Y_{T}(\omega)\right|^{2}=\sum_{\ell} \sum_{m} g\left(t_{\ell}\right) g^{*}\left(t_{m}\right) \mathrm{e}^{j \omega\left(t_{m}-t_{\ell}\right)} ; t \epsilon[-T, T)
\end{gathered}
$$

First assume that $\lambda(t)$ is known. For a given $\lambda(t)$ and conditioned on $k$ arrivals in $[-T, T)$, the joint probability density of the unordered arrival times $t_{k}=\left\{t_{1}, t_{2}, \ldots, t_{k}\right\}$ can be expressed as

$$
p_{\mathfrak{t}_{k}}\left(t_{1}, t_{2}, \ldots, t_{k} \mid k\right)=\prod_{i=1}^{k}\left(\frac{\lambda\left(t_{i}\right)}{m_{T}}\right) ; t \in[-T, T)
$$

where

$$
m_{T} \triangleq \int_{-T}^{T} \lambda(t) d t
$$

Thus,

$$
\begin{aligned}
E_{t_{k}}\left\{\left|Y_{T}(\omega)\right|^{2} \mid k\right\} & =\sum_{\ell} \sum_{m} \int_{-T}^{T} \cdots \int_{-T}^{T} g\left(t_{\ell}\right) g^{*}\left(t_{m}\right) \\
& \times \mathrm{e}^{j \omega\left(t_{m}-t_{\ell}\right)} \prod_{i=1}^{k}\left(\frac{\lambda\left(t_{i}\right)}{m_{T}}\right) d t_{1} d t_{2} \ldots d t_{k} \\
= & k \int_{-T}^{T}|g(t)|^{2} \frac{\lambda(t)}{m_{T}} d t \\
& +\frac{\left(k^{2}-k\right)}{m_{T}^{2}} \int_{-T}^{T} d t_{\ell} g\left(t_{\ell}\right) \lambda\left(t_{\ell}\right) \mathrm{e}^{-j \omega t_{\ell}} \\
& \times \int_{-T}^{T} d t_{m} g^{*}\left(t_{m}\right) \lambda\left(t_{m}\right) \mathrm{e}^{j \omega t_{m}}
\end{aligned}
$$

The first term accounts for those $k$ elements for which $\ell=m$, while the second term is due to the remaining $\left(k^{2}-k\right)$ elements of the double sum. Since for a Poisson process with rate $m_{T}, E(k)=m_{T}$ and $E\left(k^{2}-k\right)=m_{T}^{2}$, it follows from Eq. (B-5) that

$$
\begin{aligned}
E_{k}\left\{E_{\mathbf{t}_{k}}\left\{\left|Y_{T}(\omega)\right|^{2} \mid k\right\}\right\}= & \int_{-T}^{T}|g(t)|^{2} \lambda(t) d t \\
& +\left|F_{T}\{g(t) \lambda(t)\}\right|^{2}
\end{aligned}
$$

In general $\lambda(t)$ is itself a sample function of a random process; hence an additional averaging is required over the sample space of $\lambda(t)$. Thus, for doubly stochastic Poisson processes, the power spectrum is given by the expression

$$
S_{y}(\omega)=\lim _{T \rightarrow \infty} \frac{1}{2 T} E\left\{\int_{-T}^{T}|g(t)|^{2} \lambda(t) d t\right.
$$

$$
\left.+\left|F_{T}\{g(t) \lambda(t)\}\right|^{2}\right\}
$$

